\title{
Comparative analysis of anti-inflammatory activity of luteolin and luteolin-7-O-glucoside against galactosamine/lipopolysaccharide induced hepatotoxicity in mice
}

\author{
C.M. Park ${ }^{1}$ and Y.S. Song ${ }^{2}$ \\ ${ }^{1}$ Department of Clinical Laboratory Science, Dong-Eui University, Busan, Korea and ${ }^{2}$ Department of Food and Life \\ Sciences, Inje University, Gimhae, Gyeongnam, Korea
}

Luteolin is a naturally occurring flavone that reportedly has anti-inflammatory effect ${ }^{(1)}$. Flavones in plants are usually present in the form of glucosides, although occasionally they are found as aglycones. The bioavailability of flavones may differ when consumed as either aglycones or glycosides ${ }^{(2)}$. Nonetheless, numerous studies focused on the biological activity of flavonoid aglycones ${ }^{(3)}$ or that in vitro $^{(4)}$. These findings are supporting reason to compare the anti-inflammatory activity of aglycone and glucoside forms of flavones in vivo. This study was designed to compare the anti-inflammatory activity of luteolin and luteolin-7-O-glycoside in galactosamine (GalN)/lipopolysaccharide (LPS)-induced hepatoxicity.

Male ICR mice $(n=32$, 6-week-old) were divided randomly into 4 groups of 8 mice: normal control group, GalN/LPS group, luteolin and luteolin7- $O$-glucoside groups. Luteolin and luteolin-7- $O$-glycoside were given daily by gavage for $3 \mathrm{weeks}(50 \mathrm{mg} / \mathrm{kg} \mathrm{BW}$, respectively). On the last day, GalN and LPS (1 g and $10 \mu \mathrm{g} / \mathrm{kg} \mathrm{BW}$, respectively) were intraperitoneally administrated to all groups except normal control group, and animals were sacrificed $24 \mathrm{hr}$ later. Hepatoprotective effects of Luteolin and luteolin-7-O-glucoside was monitored by the measurement of serum biochemical parameters such as aspartate aminotransferase (AST) and alanine aminotransferase (ALT) and inflammatory parameters such as inducible nitric oxide synthase (iNOS), cyclooxygenase (COX-2) and their transcription factor nuclear factor $\kappa \mathrm{B}(\mathrm{NF} \kappa \mathrm{B})$ expressions. Liver tissues were also subjected to histophathological analysis.

GalN/LPS administration produced acute hepatic injury by a marked increase in serum AST and ALT activities $(p<0 \cdot 001)$. Elevated expressions of inflammatory markers such as iNOS, COX-2 and NFkB were observed $(p<0.001)$. Pretreatment of luteolin and luteolin 7- $O$-glucoside significantly inhibited the production of those biochemical parameters as well as the expressions of iNOS, COX-2 and their transcription factor NFKB $(p<0.001$ and $p<0.05$, respectively) against GalN/LPS. Luteolin more potently ameliorated expressions of inflammatory mediators than luteolin7- $O$-glucoside $(p<0.05)$ in GalN/LPS intoxicated mice. Liver histopathology showed that luteolin and luteolin7- $O$-glucoside reduced the incidence of inflammatory cell infiltration, apoptosis and necrosis induced by GalN/LPS in mice

In conclusion, luteolin and luteolin7- $O$-glucoside protect acute liver injury through the regulation of inflammatory mediators against GalN/LPS induced hepatotoxicity, although the effects of luteolin7-O-glucoside were weaker than those of luteolin.

This work was supported by the National Research Foundation of Korea (NRF) grant funded by the Korea government of MOE (No 201704340001).

1. Shanmugam S, Thangaraj R, Lima B et al. (2016) Biomedicine and Pharmacotheraphy 83, 1278-85.

2. Kano M, Takayanagi T, Harada K et al. (2006) J Nutr 136, 2291-6.

3. Kure A, Nakagawa K, Kondo M et al. (2016) J Agri Food Chem 64, 4246-54.

4. Hwang JT, Park OJ, Lee YK et al. (2011) Int J Mol Med 28, 25-31. 